and risk of type 2 diabetes mellitus (T2DM) among SLE patients.

Methods Using a population-based database that includes all residents of British Columbia, Canada, we conducted a retrospective, longitudinal cohort study of patients with incident SLE and incident antimalarial use. We established drug courses for antimalarials - defining each new course when a 90 day permissible gap had been exceeded between refills and calculating corresponding proportion days covered (PDC). Our primary exposure was adherence to antimalarials according to three categories: 1) adherent (PDC $\geq 0.90)$; 2) non-adherent $(0<\mathrm{PDC}<0.90)$; and 3$)$ discontinuer ( $\mathrm{PDC}=0$, no drug). T2DM outcomes were defined using International Classification of Disease 9th and 10th Revision Codes, and Canadian Drug Identity Codes for anti-diabetic medication (first date of either encounter). We used multivariable Cox's proportional hazards models with time-dependent variables to evaluate the association between adherence to antimalarials and risk of T2DM.

Results The study cohort included 1498 patients with incident SLE, with mean age of $44.4 \pm 14.8$ years and 1360 (90.8\%) women. Mean number of antimalarial prescriptions/courses over follow-up was 23.2 $\pm 37.7 / 2.1 \pm 1.8$, with mean course duration of $553.9 \pm 820.8$ days. Over median 4.62 years of follow-up, we recorded 140 incident cases of T2DM. After adjusting for age, gender, comorbidities, and concomitant medications, the hazard ratio (HR) for those who were adherent to antimalarials was 0.61 (95\% confidence interval [CI], 0.40 0.93) as compared to discontinuers, suggesting a protective effect of adherence to antimalarials. In contrast, the HR for those who were non-adherent was 0.78 (95\% CI 0.50 to $1.22)$ as compared to discontinuers. Sensitivity analyses involving permutations of permissible gaps (i.e. 120, 180 days) and PDC cut-off (i.e. 0.80) did not materially change our results.

Conclusions These population-based data show a protective effect of adherence to antimalarials on risk of T2DM in SLE patients. Given the effectiveness of antimalarials in treating SLE as well as additional benefits, findings emphasize the need to raise awareness, among health professionals and patients with SLE, of the importance of adherence to these therapies.

\section{BD-04 CLASSIFICATION OF SLE PATIENTS BASED ON LONGITUDINAL ASSESSMENT OF COMPLEMENT COMPONENT 3 IN RELATION TO COMPLEMENT COMPONENT 4}

David R Fernandez*, Mikhail Olferiev, Kyriakos Kirou, Dina Greenman, Kerri Merritt, Mary K Crow. Mary Kirkland Center for Lupus Research, Hospital for Special Surgery, New York, NY, USA

\subsection{6/lupus-2018-Ism.28}

Background Systemic lupus erythematosus (SLE) disease activity is characterized by tissue deposition of immune complexes and consumption of complement, which contribute to tissue injury. In clinical practice, it is common to encounter patients where either C3 or C4 is low in isolation, though the clinical implications of variation in $\mathrm{C} 3$ relative to $\mathrm{C} 4$ are unclear. Here we performed relationship-based clustering of SLE patients based on serum C3 and C4 levels to investigate if this could define distinct clinical subgroups of SLE patients.
Methods C3, C4 and other clinical and laboratory parameters were obtained from our proprietary database. A total of 151 SLE patients having an average of 38 (range 7-117) measurements of C3 or C4 were studied. To classify SLE patients based on the character of the relationship of $\mathrm{C} 3$ vs $\mathrm{C} 4$, we performed relationship-based clustering approach by defining linear fit parameters (including alpha, beta, standard error, and $p$ values) followed by hierarchical clustering. The clusters obtained were screened in terms of their dependency to clinical data using Chi square test or Fisher's exact test, as appropriate, with significance defined as $\mathrm{p}<0.05$.

Results Clustering based on multiple characteristics of the relationship between C3 and C4 identified 6 clusters of patients. Clusters 1 and 6 were small and did not have clear phenotypes. Cluster 2 and cluster 5 were both defined by strong correlations between C3 and C4 (Cluster 2 - r=0.81, $\mathrm{p}<0.00001$, Cluster $5-\mathrm{r}=0.81, \mathrm{p}=0.0016)$, though cluster 5 had a lower median C3 level (Cluster 2 C3 $=79.5$, Cluster 5 C3 $=74.5)$. Cluster 3 had higher median levels of C3 and C4 $(\mathrm{C} 3=106.0, C 4=20.6)$, and the correlation between C3 and $\mathrm{C} 4$ was far less robust $(\mathrm{r}=0.60, \mathrm{p}=0.44730)$. Cluster 4 was notable for the lowest median C3 and C4 levels $(\mathrm{C} 3=69.8, \mathrm{C} 4=12.3)$, and no significant correlation between $\mathrm{C} 3$ and C4 was present $(\mathrm{r}=0.54, \mathrm{p}=0.121143)$.

Individuals in cluster 2 were more likely to have Jaccoud arthropathy (OR 6.11, CI 1.59 to 24.47), or a history of avascular necrosis (AVN) (OR 4.38, CI 1.55 to 12.34), but less likely to have thrombocytopenia (OR 0.15 , CI 0 to 0.98 ). Cluster 5 patients were more likely to have thrombocytopenia (OR 2.78, CI 1.04 to 7.43) and less likely to have AVN (OR 0.27 , CI 0.05 to 0.99 ).

Conclusions C3 and C4 levels vary widely in SLE patients but generally fall into a few general patterns, which are associated with different clinical manifestations, and may provide novel insight into underlying biological differences between SLE patients.

Acknowledgements This work was supported by the Emerald Foundation, the Barbara Volcker Center, and the Lupus and APS Center of Excellence of the Hospital for Special Surgery.

\section{BD-05 DISCOVERY OF SERPINA3 AS A CANDIDATE URINARY BIOMARKER OF LUPUS NEPHRITIS CHRONICITY}

${ }^{1}$ Jessica L Turnier*, ${ }^{1}$ Hermine I Brunner, ${ }^{2}$ Michael Bennett, ${ }^{3}$ Ashwaq Aleed, ${ }^{4}$ Gaurav Gulati, ${ }^{5}$ Wendy D Haffey, ${ }^{1}$ Sherry Thornton, ${ }^{6}$ Michael Wagner, ${ }^{2}$ Prasad Devarajan, ${ }^{7}$ David Witte, ${ }^{5}$ Kenneth D Greis, ${ }^{6}$ Bruce Aronow. ${ }^{1}$ Rheumatology, Cincinnati Children's Hospital Medical Center; ${ }^{2}$ Nephrology and Hypertension, Cincinnati Children's Hospital Medical Center; ${ }^{3}$ Qassim University College of Medicine, Qassim, Saudi Arabia; ${ }^{4}$ Immunology, Allergy and Rheumatology; ${ }^{5}$ Cancer Biology, University of Cincinnati College of Medicine; ${ }^{6}$ Biomedical Informatics, Cincinnati Children's Hospital Medical Center, Cincinnati, OH, USA; ${ }^{7}$ Pathology and Laboratory Medicine, Cincinnati Children's Hospital Medical Center, Cincinnati, OH, USA

\subsection{6/lupus-2018-Ism.29}

Background Non-invasive biomarkers of lupus nephritis (LN) damage are needed to guide treatment decisions. Urinary proteomics has advanced as a tool for novel biomarker discovery in recent years. Specifically, isobaric tags for relative and absolute quantification (iTRAQ) is an advanced proteomics technique that quantifies and compares protein expression among samples by mass spectrometry in a single experiment. We used 
an unbiased proteomics approach to identify candidate urine biomarkers (CUBMs) predictive of LN chronicity and further pursued their validation in a larger cohort.

Methods In this cross-sectional pilot study, we selected urine collected at kidney biopsy from 20 children with varying levels of LN damage (discovery cohort) and performed proteomic analysis using iTRAQ. We identified differentially excreted proteins based on degree of LN chronicity and sought to distinguish markers exhibiting different relative expression patterns using hierarchically-clustered log10-normalized relative abundance data with linked and distinct functions by biological network analyses. For each CUBM, we performed specific enzyme-linked immunosorbent assays (ELISAs) on urine from a validation cohort $(n=41)$ and analysis of variance (ANOVA) to detect differences between LN chronicity, with LN activity adjustment. We evaluated for CUBM expression in LN biopsies with immunohistochemistry.

Results iTRAQ detected 112 proteins from urine samples in the discovery cohort, 51 of which were quantifiable in all replicates. Simple ANOVA revealed four differentially expressed, chronicity-correlated proteins $(\mathrm{p}$-values $<0.05)$. Further correlation and network analyses led us to select a total of seven CUBMs for LN chronicity: afamin (AFM), immunoglobulin heavy constant alpha 1 (IGHA1), alpha-1-antichymotrypsin (SERPINA3), transthyretin (TTR), retinol binding protein 4 (RBP4), alpha-1-acid glycoprotein, type 2 (ORM2) and transferrin (TF). In the validation cohort, urine SERPINA3 elevation was strikingly robust with respect to validation in high chronicity LN samples (3.3-fold change, p-value 0.012). Immunohistochemistry further demonstrated SERPINA3 staining in both endothelial and proximal tubular epithelial cells.

Conclusions Using advanced proteomic techniques followed by confirmation using specific ELISAs, we identified SERPINA3, a known inhibitor of neutrophil cathepsin $G$ and angiotensin II production, as a potential urine biomarker to help quantify LN damage. SERPINA3 expression may be a protective mechanism from further kidney damage. Further validation of SERPINA3 as an LN damage biomarker in an independent cohort is needed to determine its ability to guide treatment and predict prognosis.

Acknowledgements This study was supported by grants from the National Institutes of Health [P50 DK 096418, U01 AR065098, T32 AR069512, P30 AR070549] and a Lupus Foundation Career Development Award to Dr. Turnier. Mass spectrometry data were collected on a system funded through an NIH shared instrumentation grant (S10 RR027015-01; KD Greis-PI).

\section{BD-06 IDENTIFICATION OF SYSTEMIC LUPUS ERYTHEMATOSUS SUBGROUPS USING ELECTRONIC HEALTH RECORD AND GENETIC DATABASES}

${ }^{1}$ Milena A Gianfrancesco*, ${ }^{2}$ shan Paranjpe, ${ }^{1}$ Julia Kay, ${ }^{1}$ Joanne Nititham, ${ }^{1}$ Kimberly Taylor, ${ }^{1}$ Lindsey Criswell, ${ }^{2}$ Marina Sirota, ${ }^{1}$ Gabriela Schmajuk, ${ }^{1}$ Jinoos Yazdany. ${ }^{1}$ Division of Rheumatology, Department of Medicine, University of California, San Francisco; 'Institute for Computational Health Sciences, University of California, San Francisco

\subsection{6/lupus-2018-Ism.30}

Background Systemic lupus erythematosus (SLE) is a multifactorial autoimmune disease with genetic and environmental risk factors, as well as heterogeneous manifestations that encompass a wide range of disease severity. Long-term outcomes for individual patients are therefore difficult to predict, as is the scope of organ-system involvement. Little is known about why an affected individual might develop a particular SLE phenotype. A number of studies have used phenotype-mapping approaches to identify subgroups of SLE using genome-wide association studies and gene expression data; however, studies integrating the contribution of both genetic and clinical factors to identify SLE phenotypes in large, comprehensive data sources using bioinformatics analyses remain limited.

Methods We characterized subgroups of SLE patients using genetic, sociodemographic, and clinical variables from previously collected genetic cohorts and electronic health record (EHR) data for 712 individuals with SLE. Genetic data included 95 single nucleotide polymorphisms (SNPs) associated with SLE in the literature genotyped on the Immunochip platform. Variables extracted from the EHR included age, sex, race/ethnicity, age at disease onset, Charlson comorbidity index, and various disease-associated laboratory measures. Preliminary clustering was conducted using multiple correspondence analysis.

Results Approximately 90\% of SLE patients represented in genetic and EHR databases were female, and 50\% self-identified as Caucasian, 12\% Hispanic, 10\% African-American, 14\% Asian, and 14\% Other/Missing. Preliminary results showed distinct clustering by race/ethnicity amongst the 95 SNPs associated with SLE (figure 1). Further clustering and network approach analyses incorporating both genetic and clinical variables, such as laboratory measures, are ongoing and will explore whether distinct SLE subgroups can be identified.

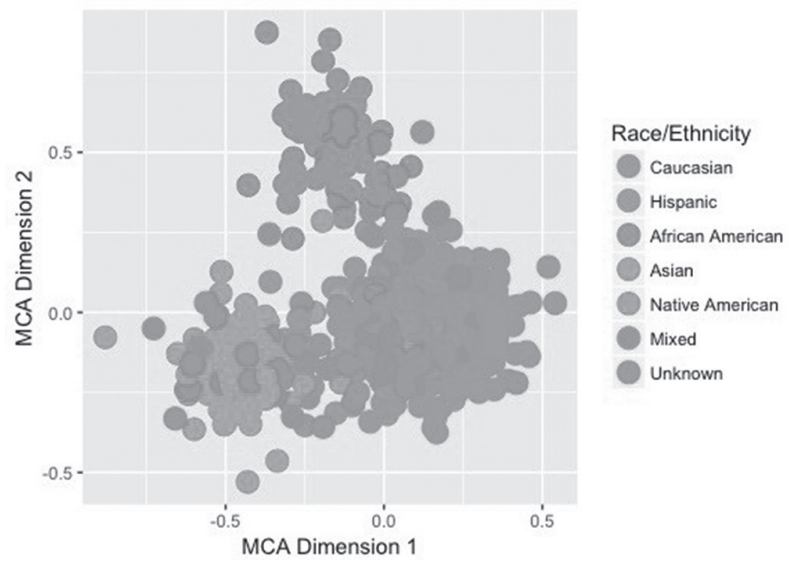

Abstract BD-06 Figure 1 Ninety-five SNPs associated with SLE demonstrate clustering by race/ethnicity

Conclusion This project is a first step towards identifying subgroups of SLE patients through clinical and genetic databases. These findings will contribute to our understanding of SLE and illustrate how combining big data in both genetics and EHR has the potential to further define this heterogeneous disease.

Acknowledgements This work was supported by NIH/NIAMS (grant number F32 AR070585 to M.A.G. and K23 AR063770 to G.S.); AHRQ [grant number R01 HS024412 to J.Y.]; and PREMIER, a NIH/NIAMS P30 Center for the Advancement of Precision Medicine in Rheumatology at UCSF (AR040155). Drs. Yazdany and Schmajuk are also supported by the Russell/ Engleman Medical Research Center for Arthritis. The content is solely the responsibility of the authors and does not necessarily represent the official views of the AHRQ or NIH. 